\title{
Retraction Note to: Chinese calligraphy practice and aggressive behaviors among children: The role of trait aggression and aggressive motivation
}

\author{
Qian Zhang ${ }^{1}$ (D) El-Lim Kim² ${ }^{2}$ JingJin Tian ${ }^{1} \cdot \mathrm{Yi} \mathrm{Cao}^{1}$ \\ @ S Springer Science+Business Media, LLC, part of Springer Nature 2021 \\ Retraction Note to: Current Psychology (2021) 40:3103-3112) \\ https://doi.org/10.1007/s12144-021-01692-w
}

The Editor-in-Chief has retracted this article because publication concerns were raised regarding several aspects of the paper, most notably the statistical treatment of the data. Post publication peer review has found that issues with the effect sizes and analysis of the data were most pronounced. The Editor therefore no longer has confidence in the accuracy of the reported data and the conclusions of the article.

Author El-Lim Kim agrees to this retraction. Authors Qian Zhang, JingJin Tian, and Yi Cao have not responded to correspondence from the editor or publisher about this retraction.

Publisher's Note Springer Nature remains neutral with regard to jurisdictional claims in published maps and institutional affiliations.

The original article can be found online at https://doi.org/10.1007/ s12144-021-01692-w

Qian Zhang

zhq@swu.edu.cn

El-Lim Kim

ellimkim@iastate.edu

JingJin Tian

9114978@qq.com

Yi Cao

2271613535@qq.com

1 Center for Studies of Education and Psychology of Ethnic Minorities in Southwest China \& Faculty of Education, Southwest University, Chongqing 400715, China

2 Department of Psychology, Iowa State University, Ames, IA 50010, USA 